\title{
The clinical spectrum of pulmonary aspergillosis
}

\author{
Chris Kosmidis, David W Denning
}

The National Aspergillosis Centre, University Hospital of South Manchester, The University of Manchester, Manchester Academic Health Science Centre, Manchester, UK

\section{Correspondence to} Dr Chris Kosmidis, The National Aspergillosis Centre, University Hospital of South Manchester, The University of Manchester, Manchester Academic Health Science Centre, Manchester M23 9LT, UK. chris.kosmidis@manchester.ac. uk

Received 8 September 2014 Revised 2 October 2014 Accepted 9 October 2014 Published Online First 29 October 2014
CrossMark

To cite: Kosmidis C Denning DW. Thorax 2015;70:270-277.

\section{ABSTRACT}

The clinical presentation of Aspergillus lung disease is determined by the interaction between fungus and host. Invasive aspergillosis develops in severely immunocompromised patients, including those with neutropenia, and increasingly in the non-neutropenic host, including lung transplant recipients, the critically ill patients and patients on steroids. A high index of suspicion is required in patients without the classical risk factors as early presentation is usually silent and nonspecific, pyrexia uncommon and timely treatment is crucial for survival. Invasive aspergillosis has also been diagnosed in normal hosts after massive exposure to fungal spores. Chronic pulmonary aspergillosis affects patients without obvious immune compromise, but with an underlying lung condition such as COPD or sarcoidosis, prior or concurrent TB or non-tuberculous mycobacterial disease. Aspergillus bronchitis may be responsible for persistent respiratory symptoms in patients with Aspergillus detected repeatedly in sputum without evidence of parenchymal Aspergillus disease, especially in patients with bronchiectasis and cystic fibrosis. Allergic bronchopulmonary aspergillosis affects patients with asthma and cystic fibrosis, and is important to recognise as permanent lung or airways damage may accrue if untreated. Changes in the classification of Aspergillus allergic lung disease have been proposed recently. Cases of extrinsic allergic alveolitis and chronic pulmonary aspergillosis have been observed after Aspergillus exposure. Asymptomatic colonisation of the respiratory tract needs close monitoring as it can lead to clinical disease especially with ongoing immunosuppression. The various syndromes should be viewed as a semicontinuous spectrum of disease and one form may evolve into another depending on the degree of ongoing immunosuppression.

\section{INTRODUCTION}

Aspergillus is a ubiquitous fungus that causes a variety of clinical syndromes. Although exposure to Aspergillus conidia through inhalation is common, only a minority of those exposed will develop lung disease. The clinical features, course and prognosis of Aspergillus infections largely depend on the degree of immune compromise of the host, although there is increasing recognition of the importance of genetics. The interplay between the pathogen and host immune dysfunction or hyperactivity determines which clinical syndrome is more likely to develop (figure 1).

On one end of the spectrum, invasive aspergillosis (IA) predominantly affects patients with profound defects in immune function, such as haematopoietic stem cell transplant (HSCT) or solid organ transplant recipients, and patients undergoing chemotherapy or taking corticosteroids. Chronic pulmonary aspergillosis (CPA) presents in a more indolent fashion, usually affecting patients with underlying lung disease, but with no or only subtle generalised immune compromise. Recently rediscovered is Aspergillus bronchitis in those with cystic fibrosis (CF), bronchiectasis especially, as well as lung transplant recipients and those ventilated in intensive therapy units. Finally, an allergic response to inhaled Aspergillus results in allergic bronchopulmonary aspergillosis (ABPA) and severe asthma with fungal sensitisation.

Aspergillus fumigatus is the most common species implicated in all pulmonary syndromes, although Aspergillus flavus is a more common cause of various forms of allergic rhinosinusitis, postoperative aspergillosis and fungal keratitis. Aspergillus terreus is a common cause of IA in some institutions and is amphotericin B resistant. Aspergillus niger is an occasional cause of IA or Aspergillus bronchitis, but is also a proportionately more common coloniser of the respiratory tract.

Several advances have been recently made towards understanding the clinical spectrum of Aspergillus infection, with improved diagnostic capability. In this review, we present the clinical syndromes of pulmonary disease caused by Aspergillus and discuss the most important issues on their management.

\section{INVASIVE ASPERGILLOSIS}

IA implies invasion of lung tissue by hyphae as demonstrated on histology. The clinical presentation is relatively rapid, ranging from days to a few weeks. It has been studied extensively in the neutropenic host; however, it has also become increasingly recognised in patients without neutropenia.

\section{Neutropenic host}

The classic risk factor for IA is neutropenia, and the likelihood of IA correlates with its duration and depth. Platelets may also be important in defence against IA, and thrombocytopenia tends to parallel neutropenia. Angioinvasion is involved in the pathogenesis in neutropenic hosts and is responsible for the higher frequency of dissemination to other organs such as skin, brain or eyes (figure 2). ${ }^{1}$ At higher risk for IA are patients undergoing allogeneic HSCT and patients with prolonged neutropenia following chemotherapy. IA in patients with neutropenia has been extensively reviewed. ${ }^{2}{ }^{3}$

\section{Non-neutropenic host}

IA occurs in a wide range of non-neutropenic hosts. The most common unifying risk factor is corticosteroid use, often prior to hospital admission. IA has been recognised in solid organ transplant recipients (particularly lung and heart-lung transplant recipients), patients with AIDS, COPD, the critically ill intensive care unit (ICU) patients, 


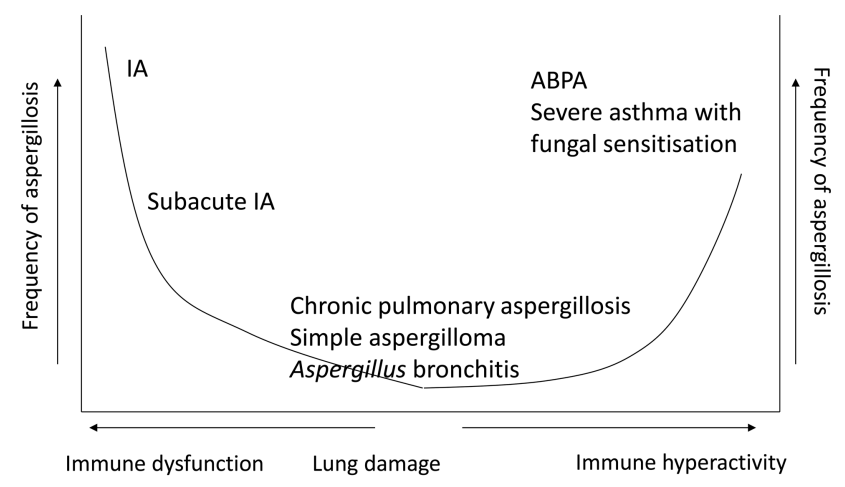

Figure 1 Interaction of Aspergillus with host. ABPA, allergic bronchopulmonary aspergillosis; IA, invasive aspergillosis.

patients with liver failure and those with chronic granulomatous disease. In these hosts, in contrast to patients with neutropenia, angioinvasion is not a common feature (figure 2). ${ }^{1}$ Fever is uncommon, and symptoms often absent until late in the course. The time course usually reflects total net immunosuppression. Many cases are relatively indolent and the condition progresses over weeks rather than over days. Lack of angioinvasion on imaging was associated with more protracted clinical course and delayed diagnosis in heart transplant recipients. ${ }^{4}$ Consequently, diagnosis is often not suspected because of the non-specific symptoms and imaging. As an example, more than half of patients with liver cirrhosis and IA where diagnosed postmortem. ${ }^{5}$ IA is the most common infection-related missed diagnosis at autopsy in ICU patients. ${ }^{6}$

\section{Lung transplant recipients}

Aspergillosis is the most common fungal infection in lung transplant recipients. In a recent surveillance study of solid organ transplant recipients, IA accounted for $44 \%$ of all fungal infections in lung transplant patients, which is much higher than that observed in other solid organ transplants, with the exception of heart transplants. ${ }^{7}$ Tracheobronchial disease is the earliest and most common manifestation, usually diagnosed when ulcerations or pseudomembranes are noted on surveillance bronchoscopy, or on diagnostic bronchoscopy for symptoms such as fever, cough and wheezing.
Invasive lung disease tends to occur later after transplant, and may be associated with dissemination to other organs.

Prophylaxis consisting of inhaled amphotericin or oral voriconazole or itraconazole is employed routinely for lung transplant patients with risk factors for development of IA. The most important factor is pre-transplant or post-transplant colonisation; the latter was an independent factor for mortality in a retrospective study. ${ }^{8}$ Other risk factors are induction with alemtuzumab or thymoglobulin, single lung transplant, cytomegalovirus infection, hypogammaglobulinaemia and rejection with use of monoclonal antibody. ${ }^{8}$ In a study of patients with CF undergoing transplantation, a positive intraoperative culture was associated with a fourfold higher risk of IA. ${ }^{10}$

\section{The critically ill}

IA is being increasingly described in the ICU setting, carries high inhospital mortality and is associated with significant costs. ${ }^{11}$ The majority of patients with IA in the ICU do not have neutropenia and have non-specific risk factors such as COPD, steroid use, multiple previous antibiotic regimens, renal or hepatic failure and diabetes. Many cases follow an episode of sepsis, probably due to so-called 'immunoparalysis'. The widespread use of steroids for COPD exacerbations may contribute to the increased rate of IA. In a multicentre study from the USA that excluded patients with the classical risk factors, $77 \%$ of critically ill patients with IA had received high-dose steroids during their hospitalisation. ${ }^{11}$ Recently, cases occurring after severe H1N1 infection and after extracorporeal membrane oxygenation use have been reported. ${ }^{12} 13$ In some cases, IA in patients admitted to the ICU has been attributed to Aspergillus contamination of air filters. ${ }^{14}$ With better supportive care that prolongs survival in critically ill patients, the incidence of IA is likely to increase.

Diagnosis of IA in the ICU is not straightforward and may be delayed by several days due to the lack of clinical suspicion. Imaging is usually non-specific, showing consolidation, patchy infiltrates, cavitation or nodules, and may be complicated by coexisting findings like atelectasis, pleural effusions or adult respiratory distress syndrome. Tracheobronchial involvement is present in a subset of patients, and may rarely be present without parenchymal disease. Bronchoscopy may reveal the presence of pseudomembranes. ${ }^{15}$
Figure 2 Pathological features and characteristics of various forms of pulmonary aspergillosis. CGD, chronic granulomatous disease; CPA, chronic pulmonary aspergillosis; GVHD, graft versus host disease; HSCT, haematopoietic stem cell transplant; IPA: invasive pulmonary aspergillosis. Adapted from Hope et al, ${ }^{1}$ with permission.

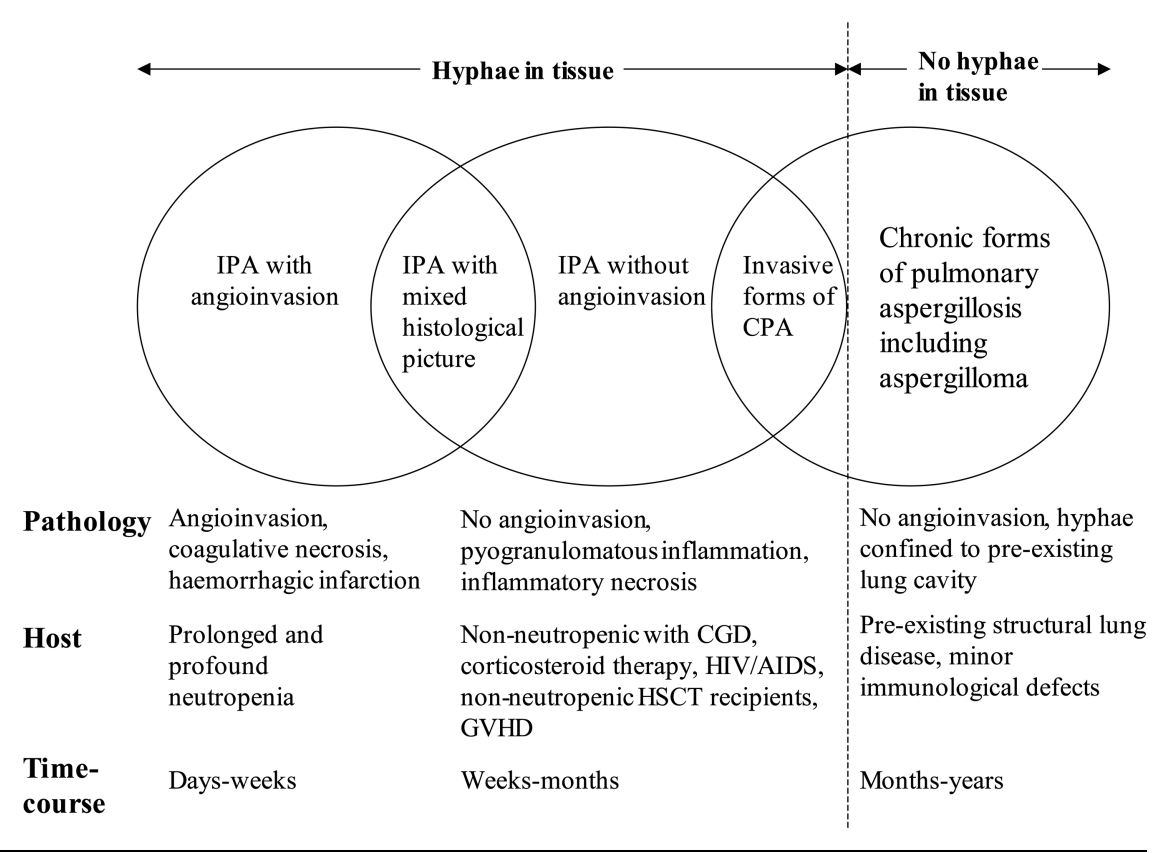




\section{Normal host}

Cases of IA have been reported in normal hosts after extensive environmental exposure to Aspergillus spores, in the form of mouldy hay, tree-bark chippings, near drowning or inhalation of dust in a mushroom factory. ${ }^{16-20}$ Arendrup et $a l^{16}$ reported two cases of IA that occurred within 1 day of gardening work involving tree-bark chippings, although symptoms may be delayed by a week or two. The source of infection may be damp or visibly mouldy. The picture is that of a severe interstitial pneumonia, or miliary type picture but cavitation may develop. This clinical presentation may be confused with extrinsic allergic alveolitis (EAA), leading to inappropriate treatment with steroids resulting in clinical deterioration.

\section{Diagnostic methods}

Although there is accumulated experience with diagnostic tests such as fungal culture, galactomannan (GM) or PCR in patients with neutropenia, less evidence exists in non-neutropenic hosts. Respiratory samples are better than blood for all tests except $\beta$-D-glucan. There are several studies that document the usefulness of bronchoalveolar lavage (BAL) GM and PCR for the diagnosis of aspergillosis in lung transplant patients. ${ }^{21} \mathrm{GM}$ sensitivity ranges from $60 \%$ to $93 \%$, depending on the cut-off value used, whereas $A$. fumigatus-specific and panfungal PCR had a sensitivity of $85 \%$ and $100 \%$, respectively. ${ }^{21}$ Both tests have a better sensitivity than that of direct microscopy and fungal culture, which is usually less than 50\%. BAL GM may also be useful as a prognostic factor, and an index $\geq 2.0$ was associated with worse prognosis in patients who were nonneutropenic. ${ }^{22}$ In contrast, serum but not BAL GM was a predictor of prognosis in patients with HSCT. $^{23}$ Sputum PCR may be useful; there are few data about sputum GM.

Among critically ill patients with COPD, BAL GM at a cut-off of 0.8 was more sensitive than fungal culture or serum GM for the diagnosis of IA. ${ }^{24}$ Evidence suggests that BAL GM may have comparable sensitivity in the non-neutropenic as in patients with neutropenia, whereas serum GM appears to be less sensitive. ${ }^{25}$ Performing more than one test (fungal culture, GM and PCR) may increase sensitivity without compromising specificity. ${ }^{26} 27$ As critically ill patients are commonly subjected to invasive diagnostic methods such as BAL, a frequent problem is the interpretation of a positive Aspergillus culture or GM test. More than one positive test is usually an indication of disease, whereas two negative respiratory sample tests almost always rule it out. In a cohort study of critically ill patients with isolation of Aspergillus, 83 out of 172 patients were classified as having definite or probable IA. ${ }^{28}$ Therefore, isolation of Aspergillus in the ICU setting must be followed by further investigations, such as a chest CT scan, and risk assessment. Use of a clinical algorithm may help distinguish between colonisation and disease. ${ }^{29}$ While IA is often radiologically non-specific, nodules, cavitation and pleural based areas of apparent infarction are distinctive. Certainly, the positive predictive value of Aspergillus isolation is higher the more immunocompromised the patient.

\section{Management}

Voriconazole is the antifungal agent of choice for treatment of IA according to almost all guidelines based on a significant mortality benefit, which is maintained in multiple real-life retrospective studies. ${ }^{8} 3031$ Although most of the data in prospective studies is from patients with haematological malignancies or HSCT, it is also considered to be the drug of choice in the non-neutropenic population. The presence of multiple comorbidities, such as renal failure in ICU patients complicates the use of voriconazole and amphotericin B, whereas liver failure makes voriconazole a less attractive option. The intravenous formulation of voriconazole contains cyclodextrin which accumulates in renal failure, but because the IA outcome is so much better with voriconazole, many authorities use voriconazole anyway. Caspofungin or micafungin offer an alternative for some patients. Voriconazole levels should be monitored early after starting therapy and a careful check for drug interactions is essential. Duration of treatment for IA in the patient who is non-neutropenic is typically a minimum of 12 weeks, subject to repeat CT scan documenting resolution of lesions and depending on the degree of ongoing immunosuppression.

\section{CHRONIC PULMONARY ASPERGILLOSIS}

CPA has only recently been recognised as a significant global health burden and its incidence appreciated. ${ }^{32}$ It is associated with significant morbidity and mortality and the optimal management strategy is not well-defined. Because of the frequency of relapse, treatment is often long-term or lifelong and therefore may be limited by intolerable side effects or the development of resistance.

CPA almost always affects patients with some form of underlying respiratory pathology that results in the formation of an air-filled cavity or bulla. Worldwide, by far the most common predisposing factor for CPA is previously treated TB. Other associations include atypical mycobacterial infection, COPD, bronchiectasis, sarcoidosis, previously treated lung cancer, ABPA and pneumothorax. Many patients have more than one underlying condition. ${ }^{33}$

The proportion of patients with CPA with previous TB varies from $15.3 \%$ in Manchester, UK to $93 \%$ in Korea. ${ }^{33}$ The persistence of lung cavities on chest X-ray 6 months after successful TB treatment was 21\% in South African gold miners and $23.3 \%$ in a North American study. ${ }^{34} 35$ Aspergillomas were detected in 14\% of patients treated for TB 1 year after sputum became negative and in $22 \%$ at 4 years, whereas Aspergillus precipitins were positive in $25 \%$ after 1 year, according to an earlier UK study. ${ }^{36} 37$ These rates are an underestimate, as surveys using CT scans have shown residual cavitation rates in excess of $30 \%$, and cavitation after multidrug resistant TB may be even higher. ${ }^{38}$ Using this information, a model predicted that the worldwide prevalence of CPA as a consequence of TB is 1.2 million people. ${ }^{32}$

In most low-resource settings, CPA may be under-recognised as a complication of previously treated $\mathrm{TB}$, and some of these patients may be misdiagnosed as having a recurrence of $\mathrm{TB}$, the two conditions having very similar symptoms. In a study performed in Uganda, more than half of patients continued to have one or more chronic symptoms such as fatigue, breathlessness, chest pain and cough, a mean of 44 months after successful treatment for TB, and this was true for HIV-positive and HIV-negative patients. $^{39}$ Interestingly, breathlessness was reported more frequently by patients living in traditional 'grass-thatch' dwellings than modern buildings, suggesting fungal exposure. ${ }^{39}$

In addition, there is a strong association between nontuberculous mycobacterial infection (NTM) and CPA. In a survey from a TB low-prevalence area (Manchester, UK), previous NTM was as common as previous TB. ${ }^{33}$ NTM may precede or present concurrently with CPA. The reason for this association is not clear, but it is likely that the underlying lung condition predisposes to infection by both pathogens and, in addition, pulmonary parenchymal damage caused by aspergillosis (cavities, bronchiectasis) increases susceptibility to infection by NTM and vice versa. A case series of patients with underlying bronchiectasis showed that patients with NTM infection were more likely to have positive Aspergillus serology than patients without NTM. ${ }^{40}$ 
The usual species from case reports and case series of NTM and CPA are Mycobacterium avium, M. xenopi, M. kansasii and M. malmoense. The usual clinical setting is failure to respond or clinical or radiological deterioration during treatment for NTM. Management of co-infection is challenging because of the prolonged courses required, the drug interactions and the often forbidding side-effect profile. In these cases, it is often elected to first treat the condition that is thought to contribute most to the patient's symptoms.

Aspergillosis complicated $2 \%$ of patients with sarcoidosis followed in a specialist clinic in the USA, based on radiology, without Aspergillus IgG serology. ${ }^{41}$ All patients had stage 4 disease on imaging, characterised mostly by upper lobe involvement, significant fibrosis and cavitation. Most case series describe poor outcomes, probably reflecting the advanced stage of sarcoidosis when CPA is diagnosed, and the need for ongoing steroid use. Although haemoptysis is rare in sarcoidosis, aspergillosis is likely the most common cause.

\section{Clinical presentation and forms of disease}

Patients with CPA are usually middle-aged, more often male, and present with constitutional symptoms (weight loss, malaise, sweats, anorexia), chronic productive cough, breathlessness, chest discomfort and occasionally haemoptysis. The latter denotes presence of an aspergilloma. Radiological findings include lung cavities with or without an aspergilloma, infiltrates, nodules and various degrees of lung or pleural fibrosis. The critical diagnostic test is Aspergillus specific IgG (or precipitins) supported by evidence of Aspergillus in sputum culture or PCR, or biopsy/aspiration.

Among patients with CPA, various patterns of presentation and evolution over time are recognised. These patterns are affected by host response, which may be modulated by various possible genetic factors and by ongoing immunosuppression.

Subjects with some degree of immunosuppression (eg, AIDS, corticosteroid treatment, diabetes mellitus, alcoholism) may present with a more rapid development of symptoms (ie, weeks as opposed to months), and with a radiological pattern of nodules, consolidation with or without a solitary cavity with thin walls, and more rapid evolution of disease over time. This pattern is termed subacute invasive aspergillosis (SAIA) or chronic necrotising pulmonary aspergillosis.

Another, more common pattern is that of slowly evolving, single or multiple lung cavities, usually with thick walls and with or without a fungus ball (aspergilloma), and with concomitant pleural fibrosis. This form is termed chronic cavitary pulmonary aspergillosis (CCPA) and appears in patients who are not overtly immunocompromised (figure 3). Despite the absence of overt immune compromise, subtle defects such as mannose-binding lectin polymorphisms, defects in surfactant, transforming growth factor- $\beta$ polymorphisms or interferon deficiency have been linked to pathogenesis of this form of CPA. ${ }^{42}$ Radiological evolution over time is slow and may take several years, and may be confused initially with other conditions like TB (figure 4). In some cases of CCPA, especially if not treated, extensive pulmonary fibrosis, characteristically unilateral, may develop over time and may involve the entire lung (figure 3). These patients are classified as having chronic fibrosing pulmonary aspergillosis.

These three forms of CPA are not separate entities and one may evolve into another over time. For example, CCPA may evolve into SAIA with ongoing immunosuppression (eg, high doses of steroids) and SAIA may eventually evolve into CCPA with antifungal therapy.

\section{Aspergilloma}

Aspergillomas are rounded conglomerates of fungal hyphae, fibrin, mucus and cellular debris that arise in pulmonary cavities, as a late manifestation of CPA. The risk of developing an aspergilloma in a cavity of $>2 \mathrm{~cm}$ in diameter is $15-20 \%$. If there is a solitary lesion, stable over months with no or minimal symptoms, a simple aspergilloma is diagnosed (figure 3). Conversely, 'complex aspergillomas' are actually CCPA, and should be managed as such, that is, medically if possible. The most serious complication of aspergilloma is haemoptysis which may be lifethreatening. The likelihood of azole resistance developing on therapy may be higher if an aspergilloma is present. ${ }^{43}$
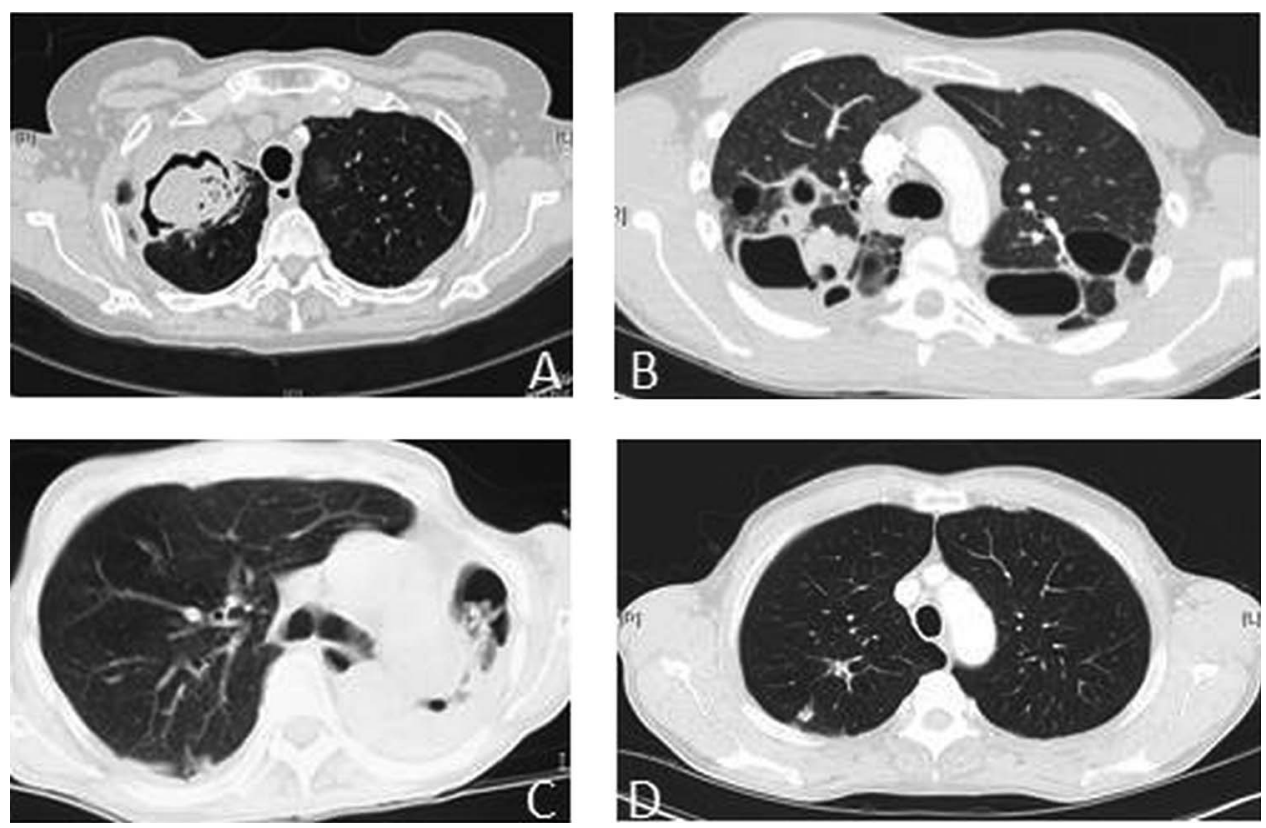

Figure 3 CT scans from patients with various forms of chronic pulmonary aspergillosis. (A) Simple aspergilloma; (B) Chronic cavitary pulmonary aspergillosis; (C) Chronic fibrosing pulmonary aspergillosis; (D) Aspergillus nodule. 


\section{Aspergillus nodule}

Aspergillus nodules are usually incidental findings on CT scan and their main clinical importance lies in their similarity to malignant lesions (figure 3). As a result, a diagnosis of Aspergillus nodule is usually made after excision biopsy. It is not clear if treatment is indicated if they are asymptomatic; serial follow-up with low dose/limited CT scans is appropriate.

\section{Management}

Management of patients with CPA is frequently complicated by the presence of comorbidities such as advanced COPD that lead to reduced lung reserve, and co-infections such as NTM. Significant health status impairment may be noted at baseline. The aims of treatment are to improve symptoms, prevent or limit haemoptysis and prevent fibrosis formation. Because of the chronicity of infection, clinical benefit is not appreciated until several weeks into the course of treatment. In addition, stopping antifungals may lead to recurrence or exacerbation. A multidisciplinary approach is needed that involves infectious disease and respiratory specialists, thoracic surgeons, nurse specialists and physiotherapists.

Azoles are the initial choice of treatment. Itraconazole is usually the first choice because of cost, followed by voriconazole and posaconazole. Several case series have shown response rates $>50 \%$ for itraconazole and voriconazole. ${ }^{4-48}$ More recently, posaconazole was also found to be effective, including a proportion of patients who failed other azoles. ${ }^{48}$ In a longitudinal study of treatment with azoles that assessed health status using the St George's Respiratory Questionnaire, approximately $40 \%$ of patients improved in 3 months, and this proportion increased at 6 months and 12 months to around $50 \% .{ }^{49}$ Among patients who remained on the same antifungal for a year, results were slightly better with posaconazole, followed by voriconazole. There was a larger proportion of patients who reported deterioration with itraconazole, but this may be attributed to side effects.
A different approach may be needed for different forms of CPA. In a prospective study of voriconazole use for 6-12 months, patients with SAIA and CCPA demonstrated different responses to treatment. ${ }^{50}$ Although clinical response was similar, complete or partial radiological improvement was seen in $41 \%$ of patients with CCPA and $79 \%$ of patients with SAIA, and serological response was more common in SAIA. Cadranel et $a l^{50}$ speculate that this may be explained by the more invasive nature of SAIA that makes the fungal organisms more readily exposed to antifungals, whereas in CCPA fungi are present within cavity walls where antifungals may not be able to penetrate. Therefore, patients with SAIA may require a shorter course of treatment compared with CCPA. In contrast, Tsubura ${ }^{51}$ showed that itraconazole can achieve adequate concentration within fungal balls. Relapse is common in CCPA if treatment is discontinued. ${ }^{52}$ The optimal management of patients that have developed significant fibrosis (chronic fibrosing pulmonary aspergillosis) is not defined, but antifungals should probably be continued indefinitely to prevent further lung damage.

For most patients with CPA, prolonged administration is usually needed, and side effects may become an important limitation to treatment. Azoles can result in liver toxicity and neuropathy, itraconazole can cause peripheral oedema, hypertension and heart failure, and voriconazole may be associated with bothersome photosensitivity. If voriconazole is continued longterm despite the presence of skin rash, precancerous lesions may develop. Posaconazole is an option for patients intolerant to or failing itraconazole or voriconazole. It has so far been available as a liquid suspension; however, a tablet formulation is now available and displays better pharmacokinetics allowing once daily administration and no food requirements.

Development of resistance is an important complication of prolonged antifungal therapy. This is not easily appreciated as sensitivity of culture is relatively low; therefore sensitivities are not often available. However, $50 \%$ of patients had evidence of
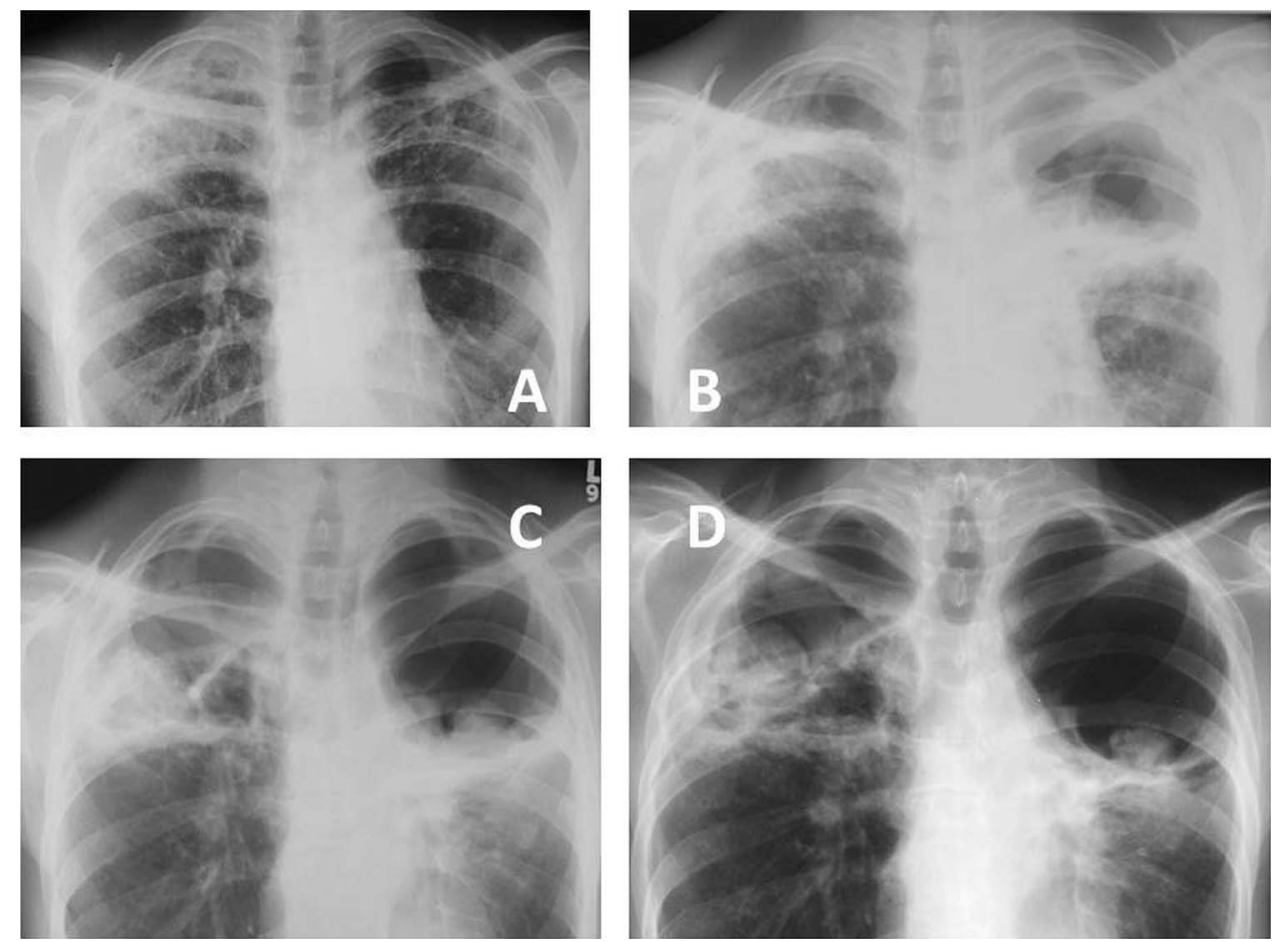

Figure 4 Serial chest X-rays of a patient with chronic cavitating pulmonary aspergillosis. (A) January 2001; (B) February 2002; (C) April 2003; (D) July 2003. 
resistance detected by molecular methods from respiratory samples. ${ }^{53}$ Patients harbouring resistant strains would be expected to respond poorly to antifungal therapy and the high rates of poor response to therapy may be explained by undetected resistance. The use of molecular methods to detect resistance mutations from respiratory samples may be used in the future to guide therapy decisions.

For patients who fail or are intolerant of azole therapy, intravenous treatment with echinocandins or amphotericin B may be used. Micafungin was found to be as effective as voriconazole in a study from Japan. ${ }^{54}$ Typically, a 3-4-week course is used, and can be repeated according to clinical response. Maintenance therapy with an azole may be used between courses. Inhaled amphotericin B is not recommended as there are no data on efficacy and may cause bronchospasm. Interferon has provided benefit in patients with impaired interferon-mediated immune responses when azole treatment had failed. ${ }^{55}$

Simple aspergillomas or complex aspergillomas with localised disease can be managed surgically in patients with an adequate respiratory reserve, although this is an option in a minority of cases. Surgical outcomes are better with simple than with complicated aspergilloma. Lobectomy is the most commonly performed procedure. ${ }^{56} 57$ A thoracoscopic approach may be indicated for some patients, and may lead to reduced hospital stay; however it may need to be converted to a full thoracotomy because of intrapleural adhesions. ${ }^{56} 58$

Although not studied, preoperative and postoperative antifungal therapy is routinely employed in simple aspergilloma. The rationale is to prevent spread of fungal organisms into the pleural cavity during the procedure, which may result in an Aspergillus empyema. If spillage of cavity contents is observed, a postoperative course of up to 12 weeks is recommended. In addition, pleural washout with amphotericin B or taurolidine $2 \%$ may be used, although the efficacy is unproven. Taurolidine was found to be active in vitro against all Aspergillus isolates from patients who underwent surgery for aspergillomas, and used for pleural decontamination during surgery. ${ }^{56}$ The risk of recurrence is about $25 \%$, probably because of a combination of underlying lung disease and genetic predisposition.

Unfortunately, relapse is common in CPA after antifungals are stopped, especially in those with more than one lobe affected. ${ }^{47} 52$ Therefore, long-term suppression rather than cure is the goal in CPA. High mortality rates are described; in a retrospective analysis, $27 \%$ died over a mean follow-up duration of 30 months, whereas a mortality of around $50 \%$ over 5 years was reported in another study. ${ }^{59}{ }^{60}$ Low body weight was found to be an important predictor of mortality in both studies.

\section{TRACHEOBRONCHIAL ASPERGILLOSIS}

Aspergillus bronchitis may account for a subset of immunocompetent patients with evidence of Aspergillus (either microbiological or serological) but without pulmonary parenchymal disease. ${ }^{61}$ These patients usually have a history of recurrent chest infections unsuccessfully managed with antibiotics and repeated isolation of Aspergillus from sputum or positive PCR. A good response to antifungals may be observed, but may be followed by relapse. In a few patients, bronchoscopy shows localised invasion of hyphae. Tracheobronchial aspergillosis in lung transplant recipients and in ICU patients is mentioned elsewhere in this review.

\section{ASPERGILLUS ALLERGIC DISEASE}

\section{Role in asthma and CF}

Aspergillus is implicated in asthma exacerbation, and increased Aspergillus spore concentrations were detected in indoor air sampled from the homes of patients with worsening symptoms of asthma. ${ }^{62}{ }^{63}$ Although inhaled fungal conidia are normally removed from the airways, defective clearance in patients with asthma and CF allows germination of conidia to hyphae, which then induce the production of proinflammatory cytokines that are responsible for the development of symptoms.

ABPA is the most common form of allergic bronchopulmonary mycosis, although other fungi can cause the syndrome. Hypersensitivity to Aspergillus is reflected by elevated Aspergillus-specific IgE levels or by a positive Aspergillus skin test. It manifests as poorly controlled asthma, and other symptoms are haemoptysis, fever, malaise and expectoration of mucous plugs. Although frequently under-recognised, this condition is important to diagnose as it can lead to permanent lung damage through the development of bronchiectasis if untreated.

Recently, diagnostic and classification criteria were reviewed by the ABPA complicating asthma working group of the International Society for Human and Animal Mycology (figure 5). ${ }^{64}$ Aspergillus-specific IgE is used as a screening test; a total $\mathrm{IgE}$ $>1000 \mathrm{IU} / \mathrm{mL}$ is then used as a diagnostic criterion for ABPA in asthma. There is no consensus regarding the cut-off values for the diagnostic serological tests; Agarwal et al $l^{65}$ recently proposed cut-off values for total IgE, Aspergillus-specific IgE and eosinophil count based on analysis of a population with ABPA in asthma in India. Cut-off values may differ in ABPA complicating asthma or CF; Baxter $e t a l^{66}$ recently proposed cut-offs for patients with CF. A subset of patients with severe asthma may not fulfil the criteria for ABPA, yet may benefit from antifungal treatment and are classified as having severe asthma with fungal sensitisation. Radiological findings in ABPA include transient infiltrates, nodules, mucus plugging and central bronchiectasis. Pleural thickening, fibrosis and aspergilloma may develop as ABPA may be complicated by CPA.

Treatment of ABPA aims to prevent progressive bronchiectasis, as well as lung fibrosis in those who will develop CPA. Corticosteroids are used at varying regimens, tapered over several weeks or months. As tapering of steroids can lead to rebound of symptoms, azoles are used in patients with frequent exacerbations in order to decrease the fungal burden and steroid dependence. Although the evidence is not very strong, antifungals have been associated with improved lung function and serological markers. ${ }^{67}$ Current experience is with itraconazole, although other azoles are also effective. ${ }^{68}$ The role of inhaled steroids is not clear in ABPA, other than in control of asthma. Nebulised amphotericin B has been used in ABPA complicating CF. Finally, omalizumab is an anti-IgE monoclonal antibody that has been used successfully in a few patients with ABPA and CF. ${ }^{69}$

\section{Extrinsic allergic alveolitis}

Cases of EAA caused by Aspergillus have been reported after exposure to mouldy hay, in bakers, in malt workers and after entry in dug wells. ${ }^{19} 7071$ As mentioned above, differential diagnosis may include IA which would necessitate antifungal therapy. The same exposure may result either to EAA or to IA in different persons. ${ }^{19}$ It is possible that massive spore exposure may result more readily to infection rather than an allergic response, or that both entities may coexist.

\section{COLONISATION}

The isolation of Aspergillus from lower respiratory samples without evidence of Aspergillus disease is defined as colonisation. The distinction between colonisation and disease relies on clinical judgement, although a stronger signal on a quantitative PCR may indicate disease rather than colonisation. The risk of clinical disease developing subsequent to colonisation depends 


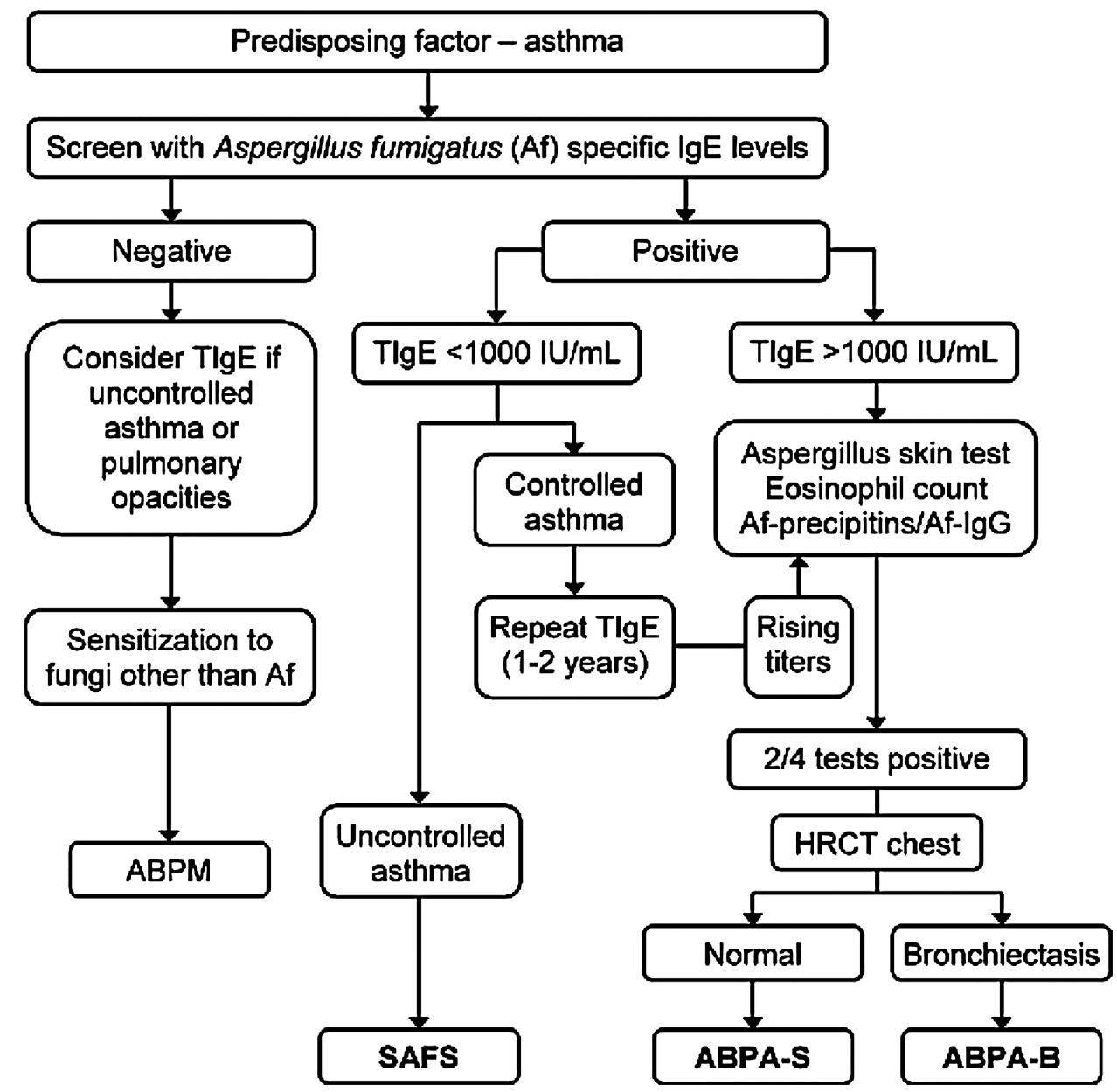

Figure 5 Classification of allergic fungal disease in asthma. ABPA, allergic bronchopulmonary aspergillosis; ABPA-B, ABPA-bronchiectasis; ABPA-S, ABPA-seropositive; ABPM, allergic bronchopulmonary mycosis; HRCT, high-resolution CT; SAFS, severe asthma with fungal sensitisation; TIgE, total $\mathrm{IgE}$. Adapted from Agarwal et $a_{1}{ }^{64}$ with permission.

on the degree of host immune compromise. For example, lung transplant recipients colonised with Aspergillus pre-transplant or in the 1st year post-transplant have a high incidence of eventually developing IA and should be offered prophylaxis with azoles or inhaled amphotericin B.

\section{CONCLUSIONS}

The various clinical syndromes caused by Aspergillus can be viewed as a continuous spectrum of disease whose manifestations are defined by the interaction between pathogen and host. One form of clinical disease may evolve into another over time depending on the degree of immune compromise of the host. Further research is necessary in order to better characterise the range and pathogenesis of infectious, allergic and saprophytic Aspergillus disease.

Competing interests None.

Provenance and peer review Not commissioned; externally peer reviewed.

\section{REFERENCES}

1 Hope WW, Walsh TJ, Denning DW. The invasive and saprophytic syndromes due to Aspergillus spp. Med Mycol 2005;43(Suppl 1):S207-38.

2 Nucci $\mathrm{M}$, Anaissie E. Fungal infections in hematopoietic stem cell transplantation and solid-organ transplantation-focus on aspergillosis. Clin Chest Med 2009:30:295-306, vii.

3 Del Bono V, Mikulska M, Viscoli C. Invasive aspergillosis: diagnosis, prophylaxis and treatment. Curr Opin Hematol 2008;15:586-93.
4 Munoz P, Vena A, Ceron I, et al. Invasive pulmonary aspergillosis in heart transplant recipients: Two radiologic patterns with a different prognosis. J Heart Lung Transplant 2014;33:1034-40.

5 Falcone M, Massetti AP, Russo A, et al. Invasive aspergillosis in patients with liver disease. Med Mycol 2011;49:406-13.

6 Winters B, Custer J, Galvagno SM Jr, et al. Diagnostic errors in the intensive care unit: a systematic review of autopsy studies. BMJ Qual Saf 2012;21:894-902.

7 Pappas PG, Alexander BD, Andes DR, et al. Invasive fungal infections among organ transplant recipients: results of the Transplant-Associated Infection Surveillance Network (TRANSNET). Clin Infect Dis 2010;50:1101-11.

8 Singh N, Husain S. Aspergillosis in solid organ transplantation. Am J Transplant 2013;13(Suppl 4):228-41.

9 Felton TW, Roberts SA, Isalska B, et al. Isolation of Aspergillus species from the airway of lung transplant recipients is associated with excess mortality. I Infect 2012:65:350-6.

10 Luong ML, Chaparro C, Stephenson A, et al. Pretransplant Aspergillus colonization of cystic fibrosis patients and the incidence of post-lung transplant invasive aspergillosis. Transplantation 2014;97:351-7.

11 Baddley JW, Stephens JM, Ji X, et al. Aspergillosis in Intensive Care Unit (ICU) patients: epidemiology and economic outcomes. BMC Infect Dis 2013;13:29.

12 Parcell BJ, B C Raju PK, et al. Invasive pulmonary aspergillosis post extracorporeal membrane oxygenation support and literature review. Med Mycol Case Rep 2014;4:12-15.

13 Wauters J, Baar I, Meersseman P, et al. Invasive pulmonary aspergillosis is a frequent complication of critically ill H1N1 patients: a retrospective study. Intensive Care Med 2012;38:1761-8.

14 Pittet D, Huguenin T, Dharan $\mathrm{S}$, et al. Unusual cause of lethal pulmonary aspergillosis in patients with chronic obstructive pulmonary disease. Am I Respir Crit Care Med 1996;154(2 Pt 1):541-4.

15 He H, Ding L, Li F, et al. Clinical features of invasive bronchial-pulmonary aspergillosis in critically ill patients with chronic obstructive respiratory diseases: a prospective study. Crit Care 2011;15:R5. 
16 Arendrup MC, O'Driscoll BR, Petersen $\mathrm{E}$, et al. Acute pulmonary aspergillosis in immunocompetent subjects after exposure to bark chippings. Scand I Infect Dis 2006;38:945-9.

17 Butler L, Brockley T, Denning D, et al. Acute Aspergillus pneumonia associated with mouldy tree bark-chippings, complicated by anti-glomerular basement membrane disease causing permanent renal failure. Med Mycol Case Rep 2013;2:125-7.

18 Shimaoka Y, Ishida T, Kawasaki S, et al. [An autopsy case of Aspergillus pneumonia after inhalation of a great deal of dust in a mushroom factory]. Nihon Kokyuki Gakkai Zasshi 2006;44:659-64.

19 Meeker DP, Gephardt GN, Cordasco EM Jr, et al. Hypersensitivity pneumonitis versus invasive pulmonary aspergillosis: two cases with unusual pathologic findings and review of the literature. Am Rev Respir Dis 1991;143:431-6.

20 Ratermann KL, Ereshefsky BJ, Fleishaker EL, et al. Fulminant invasive pulmonary aspergillosis after a near-drowning accident in an immunocompetent patient. Ann Pharmacother 2014;48:1225-9.

21 Luong ML, Clancy CJ, Vadnerkar A, et al. Comparison of an Aspergillus real-time polymerase chain reaction assay with galactomannan testing of bronchoalvelolar lavage fluid for the diagnosis of invasive pulmonary aspergillosis in lung transplant recipients. Clin Infect Dis 2011;52:1218-26.

22 Russo A, Giuliano S, Vena A, et al. Predictors of mortality in non-neutropenic patients with invasive pulmonary aspergillosis: does galactomannan have a role? Diagn Microbiol Infect Dis 2014;80:83-6.

23 Fisher CE, Stevens AM, Leisenring W, et al. The serum galactomannan index predicts mortality in hematopoietic stem cell transplant recipients with invasive aspergillosis. Clin Infect Dis 2013;57:1001-4.

24 He H, Ding L, Sun B, et al. Role of galactomannan determinations in bronchoalveolar lavage fluid samples from critically ill patients with chronic obstructive pulmonary disease for the diagnosis of invasive pulmonary aspergillosis: a prospective study. Crit Care 2012;16:R138.

25 Meersseman W, Lagrou K, Maertens J, et al. Galactomannan in bronchoalveolar lavage fluid: a tool for diagnosing aspergillosis in intensive care unit patients. Am J Respir Crit Care Med 2008;177:27-34.

26 Avni T, Levy I, Sprecher H, et al. Diagnostic accuracy of PCR alone compared to galactomannan in bronchoalveolar lavage fluid for diagnosis of invasive pulmonary aspergillosis: a systematic review. J Clin Microbiol 2012;50:3652-8.

27 Torelli R, Sanguinetti M, Moody A, et al. Diagnosis of invasive aspergillosis by a commercial real-time PCR assay for Aspergillus DNA in bronchoalveolar lavage fluid samples from high-risk patients compared to a galactomannan enzyme immunoassay. J Clin Microbiol 2011:49:4273-8.

28 Vandewoude $\mathrm{KH}$, Blot $\mathrm{SI}$, Depuydt $\mathrm{P}$, et al. Clinical relevance of Aspergillus isolation from respiratory tract samples in critically ill patients. Crit Care 2006;10:R31.

29 Blot SI, Taccone FS, Van den Abeele AM, et al. A clinical algorithm to diagnose invasive pulmonary aspergillosis in critically ill patients. Am J Respir Crit Care Med 2012;186:56-64

30 Walsh TJ, Anaissie EJ, Denning DW, et al. Treatment of aspergillosis: clinical practice guidelines of the Infectious Diseases Society of America. Clin Infect Dis 2008;46:327-60.

31 Mousset S, Buchheidt D, Heinz W, et al. Treatment of invasive fungal infections in cancer patients-updated recommendations of the Infectious Diseases Working Party (AGIHO) of the German Society of Hematology and Oncology (DGHO). Ann Hematol 2014;93:13-32.

32 Denning DW, Pleuvry A, Cole DC. Global burden of chronic pulmonary aspergillosis as a sequel to pulmonary tuberculosis. Bull World Health Organ 2011;89:864-72.

33 Smith NL, Denning DW. Underlying conditions in chronic pulmonary aspergillosis including simple aspergilloma. Eur Respir J 2011;37:865-72.

34 Sonnenberg P, Murray J, Glynn JR, et al. HIV-1 and recurrence, relapse, and reinfection of tuberculosis after cure: a cohort study in South African mineworkers. Lancet 2001;358:1687-93.

35 Hamilton CD, Stout JE, Goodman PC, et al. The value of end-of-treatment chest radiograph in predicting pulmonary tuberculosis relapse. Int I Tuberc Lung Dis 2008;12:1059-64.

36 [No authors listed]. Aspergilloma and residual tuberculous cavities - the results of a resurvey. Tubercle 1970;51:227-45.

37 [No authors listed]. Aspergillus in persistent lung cavities after tuberculosis. A report from the Research Committee of the British Tuberculosis Association. Tubercle 1968;49:1-11.

38 de Valliere S, Barker RD. Residual lung damage after completion of treatment for multidrug-resistant tuberculosis. Int J Tuberc Lung Dis 2004;8:767-71.

39 Page ID, Opwonya J, Onyachi N, et al. Chronic Pulmonary Aspergillosis complicating treated pulmonary tuberculosis in Gulu, Uganda. British Society for Medical Mycology 50th Annual Meeting. Manchester, April 27-29, 2014.

40 Kunst $\mathrm{H}$, Wickremasinghe $\mathrm{M}$, Wells $\mathrm{A}$, et al. Nontuberculous mycobacterial disease and Aspergillus-related lung disease in bronchiectasis. Eur Respir J 2006;28:352-7.

41 Pena TA, Soubani AO, Samavati L. Aspergillus lung disease in patients with sarcoidosis: a case series and review of the literature. Lung 2011;189:167-72.

42 Harrison E, Singh A, Morris J, et al. Mannose-binding lectin genotype and serum levels in patients with chronic and allergic pulmonary aspergillosis. Int J Immunogenet 2012;39:224-32.
43 Howard SJ, Cerar D, Anderson MJ, et al. Frequency and evolution of Azole resistance in Aspergillus fumigatus associated with treatment failure. Emerg Infect Dis 2009; 15:1068-76.

44 Al-Shair K, Atherton GT, Harris C, et al. Long-term antifungal treatment improves health status in patients with chronic pulmonary aspergillosis: a longitudinal analysis. Clin Infect Dis 2013;57:828-35.

45 Yoshida K, Kurashima A, Kamei K, et al. Efficacy and safety of short- and long-term treatment of itraconazole on chronic necrotizing pulmonary aspergillosis in multicenter study. J Infect Chemother 2012;18:378-85.

$46 \mathrm{Nam} \mathrm{HS}$, Jeon K, Um SW, et al. Clinical characteristics and treatment outcomes of chronic necrotizing pulmonary aspergillosis: a review of 43 cases. Int J Infect Dis 2010;14:e479-82.

47 Agarwal R, Vishwanath G, Aggarwal AN, et al. Itraconazole in chronic cavitary pulmonary aspergillosis: a randomised controlled trial and systematic review of literature. Mycoses 2013;56:559-70.

48 Felton TW, Baxter C, Moore CB, et al. Efficacy and safety of posaconazole for chronic pulmonary aspergillosis. Clin Infect Dis 2010;51:1383-91.

49 Al-shair K, Atherton GT, Kennedy D, et al. Validity and reliability of the St. George's Respiratory Questionnaire in assessing health status in patients with chronic pulmonary aspergillosis. Chest 2013;144:623-31.

50 Cadranel J, Philippe B, Hennequin C, et al. Voriconazole for chronic pulmonary aspergillosis: a prospective multicenter trial. Eur J Clin Microbiol Infect Dis 2012;31:3231-9.

51 Tsubura E. [Multicenter clinical trial of itraconazole in the treatment of pulmonary aspergilloma. Pulmonary Aspergilloma Study Group]. Kekkaku 1997;72:557-64.

52 Koyama K, Ohshima N, Suzuki J, et al. Recurrence of chronic pulmonary aspergillosis after discontinuation of maintenance treatment by antifungal triazoles. J Infect Chemother 2014;20:375-9.

53 Denning DW, Park S, Lass-Florl C, et al. High-frequency triazole resistance found In nonculturable Aspergillus fumigatus from lungs of patients with chronic fungal disease. Clin Infect Dis 2011;52:1123-9.

54 Kohno S, Izumikawa K, Ogawa K, et al. Intravenous micafungin versus voriconazole for chronic pulmonary aspergillosis: a multicenter trial in Japan. J Infect 2010;61:410-18.

55 Kelleher P, Goodsall A, Mulgirigama A, et al. Interferon-gamma therapy in two patients with progressive chronic pulmonary aspergillosis. Eur Respir J 2006;27:1307-10.

56 Farid S, Mohamed S, Devbhandari M, et al. Results of surgery for chronic pulmonary Aspergillosis, optimal antifungal therapy and proposed high risk factors for recurrence-a National Centre's experience. J Cardiothorac Surg 2013;8:180.

57 Chen QK, Jiang GN, Ding JA. Surgical treatment for pulmonary aspergilloma: a 35-year experience in the Chinese population. Interact Cardiovasc Thorac Surg 2012;15:77-80.

58 Chen QK, Chen C, Chen XF, et al. Video-assisted thoracic surgery for pulmonary aspergilloma: a safe and effective procedure. Ann Thorac Surg 2014;97:218-23.

59 Camara B, Reymond E, Saint-Raymond C, et al. Characteristics and outcomes of chronic pulmonary aspergillosis: a retrospective analysis of a tertiary hospital registry. Clin Respir J 2015;9:65-73.

60 Nakamoto K, Takayanagi N, Kanauchi T, et al. Prognostic factors in 194 patients with chronic necrotizing pulmonary aspergillosis. Intern Med 2013;52:727-34.

61 Chrdle A, Mustakim S, Bright-Thomas RJ, et al. Aspergillus bronchitis without significant immunocompromise. Ann N Y Acad Sci 2012;1272:73-85.

62 Zubairi AB, Azam I, Awan $S$, et al. Association of airborne Aspergillus with asthma exacerbation in Southern Pakistan. Asia Pac Allergy 2014;4:91-8.

63 Chen $\mathrm{CH}$, Chao HJ, Chan CC, et al. Current asthma in schoolchildren is related to fungal spores in classrooms. Chest 2014;146:123-34.

64 Agarwal R, Chakrabarti A, Shah A, et al. Allergic bronchopulmonary aspergillosis: review of literature and proposal of new diagnostic and classification criteria. Clin Exp Allergy 2013;43:850-73.

65 Agarwal R, Aggarwal AN, Garg M, et al. Cut-off values of serum IgE (total and A. fumigatus -specific) and eosinophil count in differentiating allergic bronchopulmonary aspergillosis from asthma. Mycoses 2014;57:659-63.

66 Baxter CG, Dunn G, Jones AM, et al. Novel immunologic classification of aspergillosis in adult cystic fibrosis. J Allergy Clin Immunol 2013;132:560-66 e10.

67 Moreira AS, Silva D, Ferreira AR, et al. Antifungal treatment in allergic bronchopulmonary aspergillosis with and without cystic fibrosis: a systematic review. Clin Exp Allergy 2014;44:1210-27.

68 Chishimba L, Niven RM, Cooley J, et al. Voriconazole and posaconazole improve asthma severity in allergic bronchopulmonary aspergillosis and severe asthma with fungal sensitization. J Asthma 2012;49:423-33.

69 van der Ent CK, Hoekstra H, Rijkers GT. Successful treatment of allergic bronchopulmonary aspergillosis with recombinant anti-lgE antibody. Thorax 2007:62:276-7.

70 Sharma BB, Singh S, Singh V. Hypersensitivity pneumonitis: the dug-well lung. Allergy Asthma Proc 2013;34:e59-64.

71 Gerfaud-Valentin M, Reboux G, Traclet J, et al. Occupational hypersensitivity pneumonitis in a baker: a new cause. Chest 2014;145:856-8. 